\title{
AutOPERCEPCIÓN DEL ESTADO DE ÁNIMO Y PRESENCIA DE ANSIEDAD Y DEPRESIÓN EN ESTUDIANTES UNIVERSITARIOS ${ }^{1}$ \\ Self-perception of the State of Mind and Presence of Anxiety and Depression in University Students
}

\author{
Jorge Alexander Ríos-Flórez ${ }^{2}$, Carolina Escudero-Corrales 3 , Clau- \\ dia Rocío López-Gutiérrez ${ }^{4}$, Claudia Margarita Estrada Mira ${ }^{5}$, Jona- \\ than Montes Paniagua ${ }^{6}$, Albeiro de Jesús Muñoz-Giraldo ${ }^{7}$
}

Recibido: 2019-01-08 Aceptado: 2019-03-13

Resumen: Esta investigación buscó deter-
minar la coherencia entre la autopercepción
del estado de ánimo y la presencia objetiva
de sintomatología característica de ansiedad y
depresión en estudiantes universitarios, abor-
dándose desde la perspectiva de la inteligencia
emocional y la salud mental. Se desarrolló una
investigación cuantitativa con diseńo descrip- tivo y análisis comparativo-correlacional. Con la participación de 335 estudiantes entre 18 e 30 años, 'sanos'. Se emplearon los inventarios de ansiedad y depresión de Beck y un cuestionario estándar de autopercepción. Entre otros datos se halló que, el $100 \%$ presentaba sintomatología objetiva de ansiedad. El $35 \%$ de la muestra se autopercibió con ansiedad
Para citar este artículo en APA: Ríos, J., Escudero, C., López, C., Estrada, C., Montes, J., y Muñoz, A. (2019). Autopercepción del estado de ánimo y presencia de ansiedad y depresión en estudiantes universitarios. Revista de Psicología Universidad de Antioquia, 11(1), 61-92. DOI: https://doi. org/10.17533/udea.rp.v11n1a03
Artículo resultado del macroproyecto de investigación "Psicopatología de los estados emocionales". Se expresa un agradecimiento a los participantes por la voluntad de contribuir a la generación de conocimiento y el desarrollo de propuestas de investigación encaminadas a la aplicación e intervención. A los auxiliares del proyecto por su dedicación y esfuerzo.

2 Doctor en Psicobiología, Director del Grupo de Investigación en Neurociencias Hippocampus. Universidade Federal do Rio Grande do Norte, Brasil. Correo: alexander.rios@ufrn.edu. br; https://orcid.org/0000-0002-8367-8225.

3 Psicóloga. Investigador asociado del Grupo Hippocampus, Colombia. https://orcid.org/0000-0003-2448-2983.

4 Psicóloga. Investigador asociado del Grupo Hippocampus, Colombia. https://orcid.org/0000-0002-0338-9790.

5 Estudiante de Psicología. Miembro del semillero Origen del Tecnológico de Antioquia Institución Universitaria, Medellín, Colombia.

6 Estudiante de Psicología. Miembro del semillero Origen del Tecnológico de Antioquia Institución Universitaria, Medellín, Colombia.

7 Doctorando en educación. Coordinador de los programas de Psicología y Trabajo Social del Tecnológico de Antioquia Institución universitaria, Medellín, Colombia. https://orcid. org/0000-0003-8461-7532. 
moderada y de estos sólo el 14,2 \% cumplía realmente criterios de ese nivel de ansiedad. Asimismo, $20 \%$ de los universitarios manifestó no identificar síntomas característicos de depresión en sí mismos, sin embargo, de ese porcentaje, el 31,3\% sí cumplía criterios para depresión, al igual que el $70 \%$ del total de participantes. Pese a esto, de forma general se encontró que los participantes se autopercibieron con mayores estados de ansiedad y depresión de los que realmente presentaban (variando esto por género y edad); un porcentaje menor, aunque significativo, presentó estados de ánimo patológicos mayores a los autopercibidos.

Palabras Clave: Adolescencia, afectividad, autoestima, comportamiento, salud mental.

Abstract: This research was made to determine the coherence between self-perception of the state of mind and the objective presence of symptomatology of anxiety and depression in university students, from the perspective of emotional intelligence and mental health.
A quantitative research was conducted using descriptive design and comparative-correlational analysis. 335 "healthy" students between 18 and 30 years old participated. We used the Beck anxiety and depression inventories and a standard self-perception questionnaire. Among other data, was found that 100 $\%$ presented objective anxiety symptoms. 35 $\%$ of the sample was self-perceived with moderate anxiety and of these only $14.2 \%$ really met criteria of that level of anxiety. Likewise, $20 \%$ of university students said did not identify characteristic symptoms of depression in themselves, however, of that percentage, 31.3 $\%$ did meet criteria for depression, as did $70 \%$ of the total participants. Despite this, it was generally found that the participants self-perceived with greater states of anxiety and depression than what they had (varying this by gender and age); a smaller percentage, although significant, presented pathological mood states greater than self-perceived ones.

Keywords: Adolescence, affectivity, behaviour, mental health, self-esteem.

\section{Introducción}

Dentro de los factores determinantes que facilitan la adaptación del hombre al entorno se resalta la capacidad para comprender, controlar y reconocer emociones propias y ajenas, lo que permite guiar las ulteriores cogniciones y conductas a fin de obtener resultados beneficiosos para él y su medio (Bisquerra et al., 2012; Jiménez y López-Zafra, 2014; Rodríguez, 2013). Esta habilidad, denominada Inteligencia Emocional, y acuñada por Salovey y Mayer en 1990 ha permitido comprender en la actualidad, cómo en cada individuo operan, en diferentes niveles, ciertas habilidades internas (autoconocimiento, gestión emocional, motivación) y externas (destreza social, empatía) que dan cuenta de aptitudes que favorecen o no la funcionalidad y adaptabilidad de los mismos en diferentes etapas y esferas de sus vidas, así como sus interacciones sociales (Jiménez y López-Zafra, 2009; Ríos-Flórez y Flórez-Barco, 2017); no obstante, las habilidades previamente descritas variarán según el modelo teórico en el que se haga énfasis. 
A la fecha, los modelos teóricos con mayor evidencia científica sobre este tema son, según Fernández-Berrocal, Salovey, Vera, Extremera \& Ramos (2005), a) la teoría de habilidad de Mayer y Salovey (1997) centrado en habilidades de percepción, comprensión, regulación y facilitación emocional del pensamiento; b) el modelo mixto de Bar-on (1997) que engloba habilidades intra e interpersonales, así como adaptabilidad, manejo del estrés y estado anímico general; y c) la propuesta mixta de Goleman (1996) que incluye habilidades como el autoconocimiento y manejo emocional, la automotivación, el reconocimiento emocional de los demás y el manejo de las relaciones intrapersonales.

La Inteligencia Emocional ha aportado, además, información valiosa frente a temas como la depresión y la ansiedad, así como a su prevalencia en diversos ámbitos de la vida de las personas (Goleman y Boyatzis, 2008; Bisquerra, 2006). De estos, el trastorno de depresión se ha concebido como una de las mayores problemáticas mundiales a nivel de salud pública por su alto impacto social en materia de costos, discapacidad, demanda clínica y, por ende, por sus altos índices de prevalencia (Fried, \& Nesse, 2015; González-Forteza, Hermosillo, Vacio-Muro, Peralta y Wagner, 2015; Marcus et al., 2012; Minoletti, Sepúlveda \& Horvitz-Lennon, 2012; World Health Organization [WHO], 2007). Según estudios de análisis sistemáticos realizados por autores como Murray et al., (2012) y Ferrari et al., (2013), el trastorno depresivo mayor se encuentra catalogado como la segunda patología mental más discapacitante en el mundo. De modo similar, Campo-Cabal y Gutiérrez (2001) y Pardo, Sandoval y Umbarila (2004), refieren que la prevalencia del trastorno depresivo a nivel mundial oscila entre un 8 y un $25 \%$, cifras que extrapoladas al año 2020 ubicarían a esta patología como una de las principales causas de discapacidad mental.

A su vez, para América latina se han reportado porcentajes altos, liderados por países como Chile (15.3\%), seguidos de Argentina (12\%), Perú (11 \%) y República Dominicana (9.8 \%). Particularmente en Colombia, la Organización Mundial de la Salud [OMS], en un estudio basado en cifras del año 2015, reportó que aproximadamente el $4.7 \%$ de la población presentó sintomatología depresiva, con mayor predisposición en mujeres, jóvenes y an- 
cianos, llegando a la conclusión de que el índice de prevalencia depresiva en Colombia se encuentra por encima de la media poblacional mundial (MINSALUD, 2017).

Esta patología tan incapacitante puede manifestarse en cualquier momento de la fase evolutiva del ser humano, siendo más común entre las edades de 15 a 20 años (Pérez-Álvarez y García-Montes, 2001). En Colombia, se han reportado altos índices de depresión en jóvenes universitarios comparados con la población general o con otras personas de la misma edad que no estudian, presentando mayor predisposición en adolescentes y adultos jóvenes, a su vez, consumidores de sustancias psicoactivas, mujeres y gestantes (Barraza y Medina, 2016; Kuehner, 2003).

Así mismo, los trastornos de ansiedad tienen una alta prevalencia, según Posada-Villa, Buitrago-Bonilla, Medina-Barreto y Rodríguez-Ospina (2006), quienes toman como referente el Estudio Nacional de Salud Mental-Colombia. El 19.3 \% de la población, entre 18 y 65 años, reporta haber tenido alguna vez un trastorno de ansiedad y que éste es más habitual en mujeres (Balanza, Moreno y Guerrero, 2009). Se han realizado numerosos estudios principalmente en escenarios universitarios, centrados tanto en la evaluación como en la intervención de la sintomatología característica de ansiedad (Barraza y Medina, 2016; Campo-Arias, Díaz-Martínez, Rueda-Jaimes y Barros-Bermúdez, 2005; Galli, 2005; Agudelo, Casadiegos y Sánchez, 2008), donde se ha establecido una relación directa con factores sociales, económicos, familiares y de estrés académico (Arrieta, Díaz y González, 2013; Balanza, Moreno y Guerrero, 2009; Gómez-Romero et al., 2018; Maitta, Cedeño y Escobar, 2018).

En la Universidad Industrial de Santander-Colombia se reportó en el 9\% de la población atendida en Bienestar Universitario (de 1049 estudiantes) un trastorno de ansiedad (UIS, 2005), en la Universidad Pontificia Bolivariana de Bucaramanga-Colombia (UPB) se registró un 17\% de la población consultante (327 estudiantes) con rasgos ansiosos, de los cuales el 75\% correspondían a estudiantes de Psicología (UPB, 2005); los mismos síntomas se presentaron en el $76.2 \%$ de una muestra de 973 estudiantes de universidades de Cartagena-Colombia (Arrieta et al., 2013). Por su parte, en la Universidad Cooperativa de Colombia -Sede Medellín, aplicó a 200 estudiantes la escala 
Zung, con el objetivo de determinar la prevalencia de ansiedad y su relación con determinados aspectos sociodemográficos y académicos. Los resultados muestran una prevalencia de ansiedad en el $58 \%$ de los estudiantes, con una distribución estadísticamente igual según el género y el grupo etario (Cardona-Arias et al., 2015).

Las anteriores cifras representan datos significativos a nivel de salud, por el carácter desadaptativo de la sintomatología de ansiedad y depresión, teniendo en cuenta que sus principales manifestaciones se dan a nivel corporal y las alteraciones del pensamiento (American Psychological Association, 2010), no dejando atrás los síntomas cognitivos inherentes, como la anticipación del peligro, la sensación de catástrofe, y el deterioro de las funciones neuropsicológicas, principalmente de atención y memoria (Cupul-García, Hinojosa-Calvo, Villa-Rodríguez, Herrera-Guzmán y Padrós-Blázquez, 2018; Roca, Vives, López-Navarro, García-Campayo y Gili, 2015). Asimismo, se indica que diversas formas de estrés psicológico aumentan la síntesis y liberación de cortisol (Torales et al., 2015; Cruzblanca, Lupercio, Collas y Castro, 2016); conocida como la hormona del estrés. Ese mismo tipo de estrés también influencia los desbalances neuroquímicos tras la presencia de síntomas depresivos (Sequeira y Fornaguera, 2014; Torterolo et al., 2014; Pérez-Padilla et al., 2017).

En este sentido, al establecerse el vínculo entre los estados de ánimo, los procesos electroquímicos que soportan la actividad mental y la influencia de las experiencias del individuo sobre su comportamiento y consciencia del mismo, Daniel Goleman, desde la inteligencia emocional, pone en evidencia específica la relación entre los circuitos mentales del impulso, la perseverancia y la motivación, cuando están asociados a la capacidad de ser conscientes de los propios sentimientos y comprenderlos (Goleman, 2013).

Es así que, en el ámbito educativo se han realizado diversos estudios de salud mental que buscan explicar la posible relación entre la inteligencia emocional y alteraciones en el estado de ánimo, encontrando una alta correlación entre manifestaciones de ansiedad y depresión, y una autopercepción emocional negativa de estudiantes en comparación a la población general (Azzam, Chandavarkar \& Mathews, 2007; Barraza et al., 2015; Delgado-Gómez, Gómez-Díaz, Gómez-Sánchez y Reche-García, 2019; Gómez-Romero et al., 2018). 
[66] Jorge Alexander Ríos, Carolina Escudero, Claudia Rocío López, Claudia Margarita Estrada, Jonathan Montes, Albeiro de Jesús Muñoz

Lo anterior indicaría una falencia de recursos emocionales para afrontar adecuadamente situaciones estresantes asociadas a las exigencias académicas, así como a nuevas responsabilidades, presiones familiares, económicas, sociales y cambios físicos, psicológicos, culturales y cognitivos propios de las etapas de adolescencia tardía y adultez temprana en las que se encuentran, que, al interactuar con factores predisponentes a patologías como la ansiedad y la depresión (antecedentes familiares, elección de pareja, cambios de residencia, enfermedad propia o de algún familiar, matrimonio, embarazo, inicio o pérdida laboral, muerte de un ser querido, etc.) generarían como consecuencia un deterioro global de la salud mental del estudiante (Arrieta et al., 2013; Barraza y Medina, 2016; Barraza-López, Muñoz-Navarro y Behrens-Pérez, 2017; Dávila, Ruiz, Moncada y Gallardo, 2011; Peláez et al., 2014).

De igual forma, otros estudios como los realizados por Agudelo, Casadiegos y Sánchez $(2008 ; 2009)$ reportaron una alta correlación para ansiedad y depresión en jóvenes universitarios, en asociación con factores de riesgo como consumo de alcohol y sustancias psicoactivas, ruptura de relación afectiva, pérdida de un semestre académico, niveles bajos de satisfacción y de sentido de vida, pobre control emocional, entre otros; lo que apoya los resultados expuestos en investigaciones previas centradas en este tema (Barraza y Medina, 2016; Caballero, Breso y González, 2015; Flores et al., 2007; Gallagher, 2002; Hurtado et al., 2011; Marenco, Suárez y Palacio, 2017).

Estudios como el realizado por Barraza-López, Muñoz-Navarro y Behrens-Pérez (2017), sugieren que dichas correlaciones explicarían cómo los altos niveles de depresión y ansiedad implicarían una tendencia en los estudiantes a presentar ciclos rumiativos que intensifican las autopercepciones negativas y con ello, la perpetuación de las manifestaciones clínicas. Asimismo, estos y otros autores como Pineda (2012), Ruiz, Extremera y Pineda (2014) resaltan la importancia de considerar la inteligencia emocional como un factor protector para la salud mental y física de los individuos, especialmente de los estudiantes, dado que a mayor reconocimiento, comprensión y manejo de las emociones personales, y de los otros, en situaciones adversas o estresantes, menor será la predisposición a presentar sintomatología de depresión, ansiedad o estrés, facilitando, de forma general, mayores niveles de bienestar subjetivo 
y calidad de vida (Barraza-López, Muñoz-Navarro y Behrens-Pérez, 2017; Pineda, 2012).

En este sentido, ante el panorama actual, y en consideración a la relevancia del desarrollo de planes y programas de promoción y prevención de la salud mental en ámbitos y entornos de educación superior, es válido cuestionar si los universitarios cuentan con la habilidad de reconocer sus propios estados emocionales y si son capaces o no de valorar asertivamente la magnitud de estos; destacando que, si bien la literatura permite inferir sobre la capacidad de los adolescentes y adultos jóvenes en reconocer sus estados de ánimo, se prevé la existencia de una limitación en identificar la magnitud de los síntomas característicos. Por lo anterior, la investigación aquí desarrollada se propuso determinar la coherencia entre la autopercepción del estado de ánimo y la presencia real de sintomatología característica de ansiedad y depresión en estudiantes universitarios, abordándose desde la perspectiva de la inteligencia emocional y la salud mental del adolescente y el adulto joven.

\section{Metodología}

\section{Diseño}

La investigación ejecutada siguió el modelo cuantitativo, elaborada bajo un diseño descriptivo y de análisis comparativo-correlacional, con una selección de muestra aleatoria por modelo probabilístico, atendiendo a los criterios metodológicos de investigación propuestos por Hernández, Fernández y Baptista (2014) y de participación voluntaria bajo los criterios éticos del consentimiento informado.

\section{Participantes}

La muestra estuvo constituida por 335 estudiantes (93 hombres y 242 mujeres) entre los 18 y 30 años matriculados en la Facultad de Educación y Ciencias Sociales en los programas de Psicología, Trabajo Social, Licenciatura en Preescolar y Licenciatura en Básica, de la Institución Universitaria Tecnológico de 
[68] Jorge Alexander Ríos, Carolina Escudero, Claudia Rocío López, Claudia Margarita Estrada, Jonathan Montes, Albeiro de Jesús Muñoz

Antioquia (en sus sedes Itagüí, Copacabana y Robledo). Los participantes son residentes del Valle de Aburrá (desde Barbosa hasta Caldas -Antioquia). La muestra cumplió porcentajes equitativos entre las tres sedes de la Institución. El grupo inicial fue de 398 personas, sin embargo, como criterio de exclusión se decidió que los participantes no debían tener antecedentes o alteraciones asociadas a trastornos psicológicos y/o psiquiátricos, o estar recibiendo algún tratamiento farmacológico o de psicoterapia, por lo que tras la selección se obtuvo la muestra final.

\section{Instrumentos}

El protocolo diseñado estuvo conformado por dos cuestionarios empleados para valorar el estado emocional y aquellos factores asociados a síntomas de depresión y ansiedad; como escalas psicométricas se tomaron el Inventario de Depresión de Beck - BDI (Beck, 2002a; [BDI-II]) y el Inventario de Ansiedad de Beck - BAI (Beck, 2002), además se incluyó una pregunta estandarizada para la investigación de valoración subjetiva para cada estado, en el cuestionario de datos generales (adaptada de Botelho et al., 2004), ésta hacía referencia a qué tan deprimido y ansioso (respectivamente) se sintió en los siete días previos al momento de responder el cuestionario, con calificación de 0 a 10 (se homologaron por estandarización los valores de la escala subjetiva a los rangos de las escalas objetivas a fin de facilitar su análisis y coherencia).

\section{Procedimiento}

Previo a la aplicación del protocolo se procedió a la firma de consentimiento informado, seguido de un cuestionario de datos generales en el cual se incluía la pregunta estándar de autopercepción. Posteriormente, se pidió diligenciar los inventarios de Beck; todo ello en una sesión de 20 minutos aproximadamente.

\section{Consideraciones éticas}

Esta investigación y su desarrollo metodológico se adaptó a las disposiciones legales requeridas para estudios con participantes humanos establecidas por 
el Ministerio de Salud colombiano a partir de la Resolución No. 8430 de 1993; orientándose también por la normatividad de la Ley 1090 de 2006 que formula el Código bioético y deontológico del Psicólogo, y los principios éticos de la World Medical Association en la declaración de Helsinki (WMA, 2015). Se expresó la voluntad de participación por medio del consentimiento informado tras tener conocimiento pleno del procedimiento a ser realizado, salvaguardando así la identidad, resaltando los fines netamente investigativos para el uso de la información recolectada y la posibilidad de abandonar el estudio en cualquier momento.

\section{Análisis estadisticos}

La información obtenida se analizó por medio del software estadístico SPSS versión 22, siguiendo la metodología formulada y orientados por el diseño comparativo-correlacional, se realizó la prueba Kolmogorov-Smirnov con corrección de significación de Lilliefors para identificación de normalidad de los datos y en consideración a ésta se seleccionaron (para cada criterio) los respectivos análisis paramétricos o no paramétricos; se ejecutó correlación de Spearman y Pearson, la prueba de comparación U de Mann-Whitney por la naturaleza no paramétrica de los datos, y se obtuvieron las medidas descriptivas condensados en las tablas. Los análisis de correlación para las variables BDI y BAI, se realizaron con aquellas personas (de ambos grupos) que puntuaron presencia de sintomatología característica de Depresión y Ansiedad, respectivamente.

\section{Resultados}

A continuación, se presentan inicialmente los datos descriptivos en los que se analiza la correspondencia entre la valoración subjetiva por autopercepción del estado de ánimo con los resultados objetivos de los test empleados, tanto del grupo total, como por género y edad; posteriormente, condensando en cada tabla los análisis estadísticos que ponen de manifiesto si realmente existen, o no, diferencias o relaciones significativas desde el punto de vista 
estadístico para los estados funcionales medidos; esto, tanto por comparación entre grupos como por correlación de variables y test, destacando la relevancia de aquellos datos y valores a partir de los cuales se genera la discusión y las conclusiones respectivas.

Tabla 1. Coherencia entre Autopercepción de ansiedad y Ansiedad objetiva

\begin{tabular}{|c|c|c|c|c|c|c|c|c|}
\hline & & & \multicolumn{6}{|c|}{ Ansiedad objetiva (BAI) } \\
\hline \multicolumn{3}{|l|}{ Ansiedad subjetiva } & \multicolumn{2}{|c|}{\begin{tabular}{|l|} 
Muy Baja \\
$7,88(7,01)^{2}$
\end{tabular}} & \multicolumn{2}{|c|}{ Moderada } & \multicolumn{2}{|l|}{ Severa } \\
\hline Autopercepción & Media & $\mathrm{DE}$ & \multicolumn{2}{|c|}{$7,88(7,01)^{a}$} & \multicolumn{2}{|c|}{$13,20(6,79)^{\mathrm{a}}$} & \multicolumn{2}{|c|}{$19,63(9,80)^{\mathrm{a}}$} \\
\hline Muy Baja & \multirow{2}{*}{1,63} & \multirow{2}{*}{1,23} & \multirow{2}{*}{\multicolumn{2}{|c|}{$93,30 \%$}} & \multirow{2}{*}{\multicolumn{2}{|c|}{$6,70 \%$}} & \multirow{2}{*}{\multicolumn{2}{|c|}{$0 \%$}} \\
\hline $17,90 \%$ & & & & & & & & \\
\hline Moderada & \multirow{2}{*}{5,1} & \multirow{2}{*}{0,77} & \multirow{2}{*}{\multicolumn{2}{|c|}{$85,80 \%$}} & \multirow{2}{*}{\multicolumn{2}{|c|}{$14,20 \%$}} & \multirow{2}{*}{\multicolumn{2}{|c|}{$0 \%$}} \\
\hline $35,80 \%$ & & & & & & & & \\
\hline Severa & \multirow{2}{*}{8,14} & \multirow{2}{*}{1} & \multirow{2}{*}{\multicolumn{2}{|c|}{$59,30 \%$}} & \multirow{2}{*}{\multicolumn{2}{|c|}{$33,50 \%$}} & \multirow{2}{*}{\multicolumn{2}{|c|}{$7,10 \%$}} \\
\hline $46,20 \%$ & & & & & & & & \\
\hline Total BAI & 15,22 & 9,46 & \multicolumn{2}{|l|}{$74,90 \%$} & \multicolumn{2}{|l|}{$21,80 \%$} & \multicolumn{2}{|l|}{$3,20 \%$} \\
\hline Criterio & & & $\mathrm{r} 1$ & r2 & r3 & $\mathrm{r} 4$ & 15 & $p$ \\
\hline Ansiedad Subjetiva & 5,89 & 2,60 & $0,092^{s}$ & - & $0,429^{* * \mathrm{~s}}$ & $0,511^{* * \mathrm{~s}}$ & $0,323^{* * \mathrm{~s}}$ & \multirow{2}{*}{ b } \\
\hline BAI & 15,22 & 9,47 & $0,021^{e}$ & $0,511^{\text {**s }}$ & $0,447^{* * e}$ & & $0,463^{* * e}$ & \\
\hline
\end{tabular}

Nota: $\mathrm{N}$ = 335; DE: Desviación estándar; a: Media y desviación estándar del rango en la escala BAI; r1: Correlación del criterio con la variable Edad; r2: Correlación del criterio con la variable Ansiedad subjetiva; r3: Correlación del criterio con la variable Depresión subjetiva; r4: Correlación del criterio con la prueba BAI; r5:Correlación del criterio con la prueba BDI; s: Coeficiente de correlación Rho de Spearman; e: Coeficiente de correlación de Pearson; $p$ : valor de significancia estadística de la comparación; b: No se establece comparación por tratarse de escalas diferentes; *: $p \leq 0.05 ;{ }^{* *}: p \leq 0.01 ;^{* * *}: p \leq 0.001$.

En la tabla 1 se encontró que, a nivel subjetivo un tercio de la población se ubicó en el rango Ansiedad Moderada, sin embargo, la prueba BAI refirió que únicamente el $14.2 \%$ de las personas en este rango presentaron realmente este tipo de ansiedad. En lo que respecta a la población que atribuyó puntuaciones de autopercepción ubicadas en el rango Severo (cerca de la mitad de la muestra) sólo el $7.1 \%$ de estas personas presentaron objetivamente Ansiedad Severa, según los criterios de ansiedad de la escala BAI. Así, la escala objetiva de ansiedad BAI también refirió que, de los 335 participantes de la investigación, únicamente el 3.2 \% cumplían los criterios para Ansiedad Severa. En contraste, resalta este último valor objetivo al compararse con la cantidad de personas que se ubicaron por autopercepción en el rango de Ansiedad severa (46.2\%). 
Autopercepción del estado de ánimo y presencia de ansiedad y depresión en estudiantes universitarios

En lo que respecta a las correlaciones entre las variables y los criterios considerados (tabla 1), para la muestra total, no se halló una relación de la edad (r1) con las variables subjetivas y objetivas de ansiedad, sin embargo, se encuentra que los criterios Ansiedad Subjetiva y BAI se correlaciona bidireccionalmente entre sí (r4 y r2, respectivamente). También se halló una correspondencia de estos dos criterios con la escala de Depresión Subjetiva (r3) y la escala objetiva (r5); las anteriores correlaciones se establecieron con valor de $p \leq 0.01$. Estas relaciones son directamente proporcionales, es decir, a mayor atribución de puntaje en el criterio Ansiedad Subjetiva, mayor puntuación en los inventarios objetivos de ansiedad y depresión, y en la autopercepción de sintomatología depresiva.

Tabla 2. Coherencia entre Autopercepción de depresión y Depresión objetiva

\begin{tabular}{|c|c|c|c|c|c|c|c|c|}
\hline & & & \multicolumn{6}{|c|}{ Depresión objetiva (BDI) } \\
\hline \multicolumn{3}{|c|}{ Depresión subjetiva } & Ausente & Mínima & Leve & Moderada & \multicolumn{2}{|c|}{ Severa } \\
\hline Autopercepción & Media & DE & $3,94(4,60)^{\mathrm{a}}$ & $5,58(4,47)^{a}$ & $7,91(4,99)^{\mathrm{a}}$ & $11,37(6,01)^{\mathrm{a}}$ & \multicolumn{2}{|c|}{$15,66(6,91)^{\mathrm{a}}$} \\
\hline Ausente & \multirow{2}{*}{0} & \multirow{2}{*}{0} & \multirow{2}{*}{$68,60 \%$} & \multirow{2}{*}{$25,30 \%$} & \multirow{2}{*}{$4,50 \%$} & \multirow{2}{*}{$1,50 \%$} & \multirow{2}{*}{\multicolumn{2}{|c|}{$0 \%$}} \\
\hline $20 \%$ & & & & & & & & \\
\hline Mínima & \multirow{2}{*}{1,71} & \multirow{2}{*}{0,46} & \multirow{2}{*}{$41,90 \%$} & \multirow{2}{*}{$53,20 \%$} & \multirow{2}{*}{$4,80 \%$} & \multirow{2}{*}{$0 \%$} & \multirow{2}{*}{\multicolumn{2}{|c|}{$0 \%$}} \\
\hline $18,50 \%$ & & & & & & & & \\
\hline Leve & \multirow{2}{*}{3,58} & \multirow{2}{*}{0,50} & \multirow{2}{*}{$27,70 \%$} & \multirow{2}{*}{$55,30 \%$} & \multirow{2}{*}{$13,80 \%$} & \multirow{2}{*}{$3,10 \%$} & \multirow{2}{*}{\multicolumn{2}{|c|}{$0 \%$}} \\
\hline $19,40 \%$ & & & & & & & & \\
\hline Moderada & \multirow{2}{*}{5,91} & \multirow{2}{*}{0,86} & \multirow{2}{*}{$10,30 \%$} & \multirow{2}{*}{$47,40 \%$} & \multirow{2}{*}{$36,10 \%$} & \multirow{2}{*}{$4,10 \%$} & \multirow{2}{*}{\multicolumn{2}{|c|}{$2,00 \%$}} \\
\hline $28,90 \%$ & & & & & & & & \\
\hline Severa & \multirow{2}{*}{8,48} & \multirow{2}{*}{0,76} & \multirow{2}{*}{$6,80 \%$} & \multirow{2}{*}{$29,50 \%$} & & & & \\
\hline $13,10 \%$ & & & & & $36,30 \%$ & $20,40 \%$ & $6,90 \%$ & \\
\hline Total BDI & 8,7 & 6,64 & $30,70 \%$ & $43,30 \%$ & $19,70 \%$ & $4,70 \%$ & $1,50 \%$ & \\
\hline Criterio & & & $\mathrm{r} 1$ & r2 & r3 & r4 & $\mathrm{r} 5$ & $\mathrm{p}$ \\
\hline Depresión Subjetiva & 3,84 & 2,87 & $0,069^{\mathrm{e}}$ & $0,429^{* * \mathrm{~s}}$ & - & $0,447^{* * \mathrm{e}}$ & $0,602^{* * e}$ & \\
\hline BDI & 8,7 & 6,64 & $-0,011^{s}$ & $0,323^{* * \mathrm{~s}}$ & $0,602^{* *_{e}}$ & $0,463^{* *_{e}}$ & - & \\
\hline
\end{tabular}

Nota: N= 335; DE: Desviación estándar; a: Media y desviación estándar del rango en la escala BDI; r1: Correlación del criterio con la variable Edad; r2: Correlación del criterio con la variable Ansiedad subjetiva; r3: Correlación del criterio con la variable Depresión subjetiva; r4: Correlación del criterio con la prueba BAI; r5:Correlación del criterio con la prueba BDI; s: Coeficiente de correlación Rho de Spearman; e: Coeficiente de correlación de Pearson; $p$ : valor de significancia estadística de la comparación; b: No se establece comparación por tratarse de escalas diferentes; ${ }^{*}: p \leq 0.05 ;{ }^{* *}: p \leq 0.01 ;{ }^{* *}: p \leq 0.001$. 
[72] Jorge Alexander Ríos, Carolina Escudero, Claudia Rocío López, Claudia Margarita Estrada, Jonathan Montes, Albeiro de Jesús Muñoz

Por su parte, la tabla 2 refiere que una quinta parte de la muestra total, a partir de las puntuaciones de la escala subjetiva, se ubicaron en el rango Ausente de depresión; sin embargo, las puntuaciones objetivas de las personas ubicadas en este rango indicaron que un porcentaje significativo cumplía criterios de la escala BDI para Depresión minima, y realmente el $1.5 \%$ presentaba Depresión moderada. De los 335 participantes obtuvieron puntuaciones que los ubicaban en el rango minimo de Autopercepción de depresión (18.5\%) cerca de la mitad de esta población obtuvo coherencia con el nivel Minimo de depresión de la escala BDI. En lo que respecta al rango de autopercepción leve sólo el 13.8 \% cumplió realmente criterios de depresión leve en la escala BDI, el $3.1 \%$ presentó depresión moderada en la escala objetiva y el restante de la población con Depresión subjetiva leve cumplían criterios que los ubicaban objetivamente en Depresión ausente o minima, según el BDI.

De acuerdo con lo reportado en la tabla 2, destaca que, de los participantes ubicados en el rango Moderado (28.9 \%) de la Escala de Depresión subjetiva el 2 \% llegaba a presentar clínicamente Depresión severa; los demás, presentaban síntomas de depresión menores al autopercibido. En relación con los resultados totales del BDI, se encontró que, de los 335 participantes, la mayoría presentó Depresión mínima (43.3\%) y el 1.5 \% cumplió criterios de Depresión severa.

Ahora bien, en lo que respecta a la relación entre los criterios de la tabla (2) con las escalas subjetivas y objetivas, se halló que, el criterio Depresión subjetiva se correlaciona con la escala objetiva $B D I$, bidireccionalmente ( $\mathrm{r} 5 \mathrm{y} \mathrm{r} 3$, respectivamente); en ambos casos, esta relación es directamente positiva, es decir, a mayores puntuaciones en una escala, mayores serán en la otra. Con un nivel de significancia estadística de $p \leq 0.01$. De igual forma, y con un mismo valor estadístico, estos dos criterios se correlacionaron con la escala subjetiva (r2) y objetiva (r4) de ansiedad. 
Autopercepción del estado de ánimo y presencia de ansiedad y depresión en estudiantes universitarios

Tabla 3. Coherencia entre Autopercepción de ansiedad y Ansiedad objetiva por Género

\begin{tabular}{|c|c|c|c|c|c|c|c|}
\hline \multirow[t]{2}{*}{ Rango } & \multirow[t]{2}{*}{ Género } & \multicolumn{3}{|c|}{ Ansiedad Subjetiva } & \multicolumn{3}{|c|}{ Ansiedad objetiva (BAI) } \\
\hline & & Media & $\mathrm{DE}$ & $p^{U}$ & M & $\mathrm{DE}$ & $p^{U}$ \\
\hline & Hombres & 5,51 & 2,65 & \multirow{2}{*}{0,106} & 15,06 & 9,92 & \multirow{2}{*}{0,247} \\
\hline & Mujeres & 6,03 & 2,58 & & 15,28 & 9,31 & \\
\hline \multirow{2}{*}{ Muy Baja } & Hombres & \multicolumn{3}{|l|}{$23,60 \%$} & \multirow{2}{*}{\multicolumn{3}{|c|}{$\begin{array}{l}70,90 \% \\
76,40 \%\end{array}$}} \\
\hline & Mujeres & \multicolumn{3}{|l|}{$15,70 \%$} & & & \\
\hline \multirow{2}{*}{ Moderada } & Hombres & \multicolumn{3}{|l|}{$36,50 \%$} & \multicolumn{3}{|l|}{$25,80 \%$} \\
\hline & Mujeres & \multicolumn{3}{|l|}{$35,50 \%$} & \multicolumn{3}{|l|}{$20,20 \%$} \\
\hline \multirow{2}{*}{ Severa } & Hombres & \multicolumn{3}{|l|}{$39,80 \%$} & \multicolumn{3}{|l|}{$3,20 \%$} \\
\hline & Mujeres & $48,80 \%$ & & & \multicolumn{3}{|l|}{$3,30 \%$} \\
\hline Criterio & & $\mathrm{r} 1$ & $\mathrm{r} 2$ & r3 & $\mathrm{r} 4$ & r5 & \\
\hline \multirow{2}{*}{ Ansiedad Subjetiva } & Hombres & $-0,033^{e}$ & - & $0,437^{* * \mathrm{~s}}$ & $0,477^{* * \mathrm{~s}}$ & $0,267^{* * \mathrm{~s}}$ & \\
\hline & Mujeres & $0,134^{* e}$ & - & $0,418^{* * \mathrm{~s}}$ & $0,526^{* * \mathrm{~s}}$ & $0,347^{* * \mathrm{~s}}$ & \\
\hline \multirow{2}{*}{ BAI } & Hombres & $-0,006^{s}$ & $0,477^{* * s}$ & $0,510^{* *_{e}}$ & - & $0,485^{* * e}$ & \\
\hline & Mujeres & $0,030^{e}$ & $0,526^{* * s}$ & $0,426^{* * e}$ & - & $0,468^{* * s}$ & \\
\hline
\end{tabular}

Nota: $\mathrm{N}$ Hombres= 93; N Mujeres= 242; r1: Correlación del criterio con la variable Edad; r2: Correlación del criterio con la variable Ansiedad subjetiva; r3: Correlación del criterio con la variable Depresión subjetiva; r4: Correlación del criterio con la prueba BAI; r5:Correlación del criterio con la prueba BDI; s: Coeficiente de correlación Rho de Spearman; e: Coeficiente de correlación de Pearson; $p$ : valor de significancia estadística de la comparación; U: Prueba estadística U de Mann-Whitney; ${ }^{*}: p \leq 0.05$; ${ }^{* *}: p \leq 0.01$; ***: $p \leq 0.001$.

En los análisis comparativos de la tabla 3, se encuentra que, los hombres asignan mayores puntuaciones que los ubican en autopercepción Muy baja de Ansiedad, que las mujeres. Por su parte, entre el grupo de los hombres en el rango de Ansiedad subjetiva Moderada únicamente una cuarta parte cumplían criterios reales para este nivel, según la escala objetiva BAI. En lo que refiere a las mujeres, el rango moderado de Ansiedad subjetiva moderada fue ocupado por el $35.5 \%$ de éstas, aunque realmente sólo una quinta parte de las mujeres presentó Ansiedad moderada en la Escala BAI. Resalta que un porcentaje alto de hombres y mujeres manifestaron puntuaciones de Ansiedad subjetiva severa, pese a esto, la escala objetiva arrojó que menos del $4 \%$ de estos se ubicaban en este nivel de ansiedad. Aun cuando la cantidad de hombres y mujeres no es 
[74] Jorge Alexander Ríos, Carolina Escudero, Claudia Rocío López, Claudia Margarita Estrada, Jonathan Montes, Albeiro de Jesús Muñoz

equitativa entre grupos, los análisis de significancia presentados en la tabla $(p)$ indican que no hay diferencias estadísticas al compararlos por género en estas escalas (subjetiva $=0,106$; objetiva $=0,247$ ), por lo que el comportamiento de las variables es similar entre hombres y mujeres, aun cuando, en promedio, las mujeres asignan mayores puntuaciones en autopercepción de ansiedad y obtienen mayores puntuaciones en la escala objetiva.

De otro lado, se encontró que, en las mujeres, el criterio Ansiedad Subjetiva se correlaciona con la edad positivamente $(\mathrm{r} 1)$, donde a mayor edad, mayores puntuaciones subjetivas asignadas a los síntomas de ansiedad (a nivel estadístico de $p \leq 0.05$ ). Este criterio se correlacionó para ambos géneros con la escala objetiva de ansiedad BAI (r4) y con las dos escalas de depresión ( $\mathrm{r} 3$ y r5), de forma directamente proporcional. Lo mismo se establece para el criterio BAI en las correlaciones con las escalas, principalmente con la escala subjetiva (r2); lo cual demuestra una correspondencia entre las puntuaciones asignadas por autopercepción y la medición objetiva del inventario, con valor de $p \leq 0.01$.

Tabla 4. Coherencia entre Autopercepción de depresión y Depresión objetiva por Género

\begin{tabular}{|c|c|c|c|c|c|c|c|}
\hline \multirow[t]{2}{*}{ Rango } & \multirow[t]{2}{*}{ Género } & \multicolumn{3}{|c|}{ Depresión Subjetiva } & \multicolumn{3}{|c|}{ Depresión objetiva (BDI) } \\
\hline & & Media & $\mathrm{DE}$ & $p^{U}$ & M & $\mathrm{DE}$ & $p^{U}$ \\
\hline & Hombres & 3,15 & 2,93 & \multirow{2}{*}{$0,006^{* *}$} & 8,28 & 7,14 & \multirow{2}{*}{0,784} \\
\hline & Mujeres & 4,1 & 2,82 & & 8,87 & 6,44 & \\
\hline \multirow{2}{*}{ Ausente } & Hombres & \multicolumn{3}{|c|}{$31,20 \%$} & \multicolumn{3}{|c|}{$37,60 \%$} \\
\hline & Mujeres & \multicolumn{3}{|c|}{$15,70 \%$} & \multicolumn{3}{|c|}{$28,10 \%$} \\
\hline \multirow{2}{*}{ Mínima } & Hombres & \multicolumn{3}{|c|}{$18,20 \%$} & \multicolumn{3}{|c|}{$39,80 \%$} \\
\hline & Mujeres & \multicolumn{3}{|c|}{$18,60 \%$} & \multicolumn{3}{|c|}{$44,60 \%$} \\
\hline \multirow{2}{*}{ Leve } & Hombres & \multicolumn{3}{|c|}{$18,20 \%$} & \multicolumn{3}{|c|}{$16,10 \%$} \\
\hline & Mujeres & \multicolumn{3}{|c|}{$19,80 \%$} & \multicolumn{3}{|c|}{$21,00 \%$} \\
\hline \multirow{2}{*}{ Moderada } & Hombres & \multicolumn{3}{|c|}{$21,50 \%$} & \multicolumn{3}{|c|}{$3,20 \%$} \\
\hline & Mujeres & \multicolumn{3}{|c|}{$31,80 \%$} & \multicolumn{3}{|c|}{$5,30 \%$} \\
\hline \multirow{2}{*}{ Severa } & Hombres & \multicolumn{3}{|c|}{$10,80 \%$} & \multicolumn{3}{|c|}{$3,20 \%$} \\
\hline & Mujeres & \multicolumn{3}{|c|}{$14,00 \%$} & \multicolumn{3}{|c|}{$0,90 \%$} \\
\hline
\end{tabular}


Autopercepción del estado de ánimo y presencia de ansiedad y depresión en estudiantes universitarios

\begin{tabular}{|l|l|l|l|l|l|l|}
\hline Criterio & & $\mathrm{r} 1$ & $\mathrm{r} 2$ & $\mathrm{r} 3$ & $\mathrm{r} 4$ & $\mathrm{r} 5$ \\
\hline \multirow{2}{*}{$\begin{array}{l}\text { Depresión } \\
\text { Subjetiva }\end{array}$} & Hombres & $-0,077^{\mathrm{s}}$ & $0,437^{* * \mathrm{~s}}$ & - & $0,510^{* * e}$ & $0,604^{* *_{e}}$ \\
\cline { 2 - 7 } & Mujeres & $0,116^{\mathrm{e}}$ & $0,418^{* * \mathrm{~s}}$ & - & $0,426^{* * e}$ & $0,603^{* * e}$ \\
\hline \multirow{2}{*}{ BDI } & Hombres & $0,040^{\mathrm{e}}$ & $0,267^{* * \mathrm{~s}}$ & $0,604^{* * e}$ & $0,485^{* * e}$ & - \\
\cline { 2 - 7 } & Mujeres & $-0,018^{\mathrm{e}}$ & $0,347^{* * s}$ & $0,603^{* * e}$ & $0,468^{* * \mathrm{~s}}$ & - \\
\hline
\end{tabular}

Nota: $\mathrm{N}$ Hombres= 93; $\mathrm{N}$ Mujeres= 242; r1: Correlación del criterio con la variable Edad; r2: Correlación del criterio con la variable Ansiedad subjetiva; r3: Correlación del criterio con la variable Depresión subjetiva; r4: Correlación del criterio con la prueba BAI; r5:Correlación del criterio con la prueba BDI; s: Coeficiente de correlación Rho de Spearman; e: Coeficiente de correlación de Pearson; $p$ : valor de significancia estadística de la comparación; U: Prueba estadística U de Mann-Whitney; ${ }^{*}: p \leq 0.05 ;{ }^{* *}: p \leq 0.01$; ${ }^{* * *}: p \leq 0.001$.

En la tabla 4 se observa que el porcentaje de personas en los rangos de Depresión moderada y severa de la Escala subjetiva fue superior a los que realmente cumplían criterios para estos mismos rangos en el BDI; no obstante, para los demás rangos, el porcentaje objetivo tanto de hombres como de mujeres fue mayor al reportado en Depresión subjetiva por autopercepción.

Resalta en la tabla 4, que el $18 \%$ tanto de hombres como de mujeres asignaron puntuaciones que los ubicaron en el rango de Depresión minima por autopercepción, pese a esto, el porcentaje real de los que cumplían criterios para este rango en la Escala objetiva fue más del doble, para ambos géneros. En contraste a esto, el $3.2 \%$ de los hombres presentó Depresión moderada en la Escala objetiva, pese a que fue el $21.5 \%$ de estos los que asignaron puntuaciones de autopercepción de depresión moderada. Por otra parte, el $14 \%$ de las mujeres se autopercibieron con niveles de Depresión severa, aunque menos del $1 \%$ del total de mujeres cumplieron criterios objetivos para este rango de depresión.

Un dato que destaca en la tabla 4 es el de la comparación entre hombres y mujeres en la escala de Depresión Subjetiva. Estadísticamente se encontró que las mujeres obtuvieron mayores puntuaciones por autopercepción de depresión $(p \leq 0.01)$, sin embargo, la medición de Depresión objetiva no se encontraron diferencias significativas entre los géneros. De otro lado, en lo que respecta a las correlaciones, se halló una correspondencia bidireccional entre los criterios de Depresión subjetiva y BDI ( $\mathrm{r} 5$ y r3, respectivamente), tanto para hombres como para mujeres; esta relación es directamente proporcional, 
indicando que, a mayores puntuaciones en el criterio, mayores puntuaciones en la escala correlacionada. De igual forma estos dos criterios se relacionaron con las escalas de ansiedad, y se encontró que, tanto en hombre como mujeres los criterios de Depresión subjetiva y $B D I$ se correlacionaron positiva y significativamente con la edad ( $\mathrm{r} 1)$, es decir, a mayor edad mayores puntuaciones en las dos escalas $(p \leq 0.01)$.

Tabla 5. Coherencia entre Autopercepción de ansiedad y Ansiedad objetiva por edad

\begin{tabular}{|c|c|c|c|c|c|c|c|c|c|c|}
\hline & & & & \multicolumn{7}{|c|}{ Ansiedad objetiva (BAI) } \\
\hline & & & & $<24$ & \multicolumn{2}{|c|}{$8,15(7,02)^{\mathrm{a}}$} & \multicolumn{2}{|c|}{$13,11(6,65)^{\mathrm{a}}$} & \multicolumn{2}{|c|}{$19,76(9,68)^{a}$} \\
\hline \multicolumn{4}{|c|}{ Ansiedad subjetiva } & $>23$ & \multicolumn{2}{|c|}{$6,13(7,12)^{a}$} & \multicolumn{2}{|c|}{$13,80(7,96)^{\mathrm{a}}$} & \multicolumn{2}{|c|}{$18,93(10,59)^{\mathrm{a}}$} \\
\hline \multicolumn{2}{|c|}{ Autopercepción } & Edad & Media & $\mathrm{DE}$ & \multicolumn{2}{|c|}{ Muy Baja } & \multicolumn{2}{|c|}{ Moderada } & \multicolumn{2}{|c|}{ Severa } \\
\hline \multirow{2}{*}{ Muy Baja } & $18,10 \%$ & $<24$ & 1,6 & 1,22 & \multicolumn{2}{|l|}{$92,30 \%$} & \multicolumn{2}{|l|}{$7,70 \%$} & \multicolumn{2}{|l|}{$0 \%$} \\
\hline & $16,60 \%$ & $>23$ & 1,88 & 1,35 & \multicolumn{2}{|l|}{$100 \%$} & \multicolumn{2}{|l|}{$0 \%$} & \multicolumn{2}{|l|}{$0 \%$} \\
\hline \multirow{2}{*}{ Moderada } & $36,60 \%$ & $<24$ & 5,07 & 0,77 & \multicolumn{2}{|l|}{$85,70 \%$} & \multicolumn{2}{|l|}{$14,30 \%$} & \multicolumn{2}{|l|}{$0 \%$} \\
\hline & $31,30 \%$ & $>23$ & 5,33 & 0,72 & \multicolumn{2}{|l|}{$86,60 \%$} & \multicolumn{2}{|l|}{$13,30 \%$} & \multicolumn{2}{|l|}{$0 \%$} \\
\hline \multirow{2}{*}{ Severa } & $45,30 \%$ & $<24$ & 8,12 & 1 & \multicolumn{2}{|l|}{$57,70 \%$} & \multicolumn{2}{|l|}{$35,40 \%$} & \multicolumn{2}{|l|}{$6,90 \%$} \\
\hline & $52,10 \%$ & $>23$ & 8,28 & 1,02 & \multicolumn{2}{|l|}{$68 \%$} & \multicolumn{2}{|l|}{$24 \%$} & \multicolumn{2}{|l|}{$8 \%$} \\
\hline \multirow{2}{*}{ Total BAI } & $100 \%$ & $<24$ & 13,67 & 5,78 & \multicolumn{2}{|l|}{$74,20 \%$} & $22,60 \%$ & & $3,10 \%$ & \\
\hline & $100 \%$ & $>23$ & 12,95 & 8,56 & $79,10 \%$ & & $16,70 \%$ & & $4,20 \%$ & \\
\hline Criterio & & & & & $\mathrm{r} 1$ & $\mathrm{r} 2$ & r3 & $\mathrm{r} 4$ & r5 & $p^{U}$ \\
\hline Ansiedad & & $<24$ & 5,82 & 2,61 & $0,066^{s}$ & - & $0,438^{* * \mathrm{~s}}$ & $0,516^{* * \mathrm{~s}}$ & $0,633^{* * \mathrm{~s}}$ & \\
\hline$>23$ & & 6,29 & 2,58 & $0,025^{\mathrm{e}}$ & - & $0,378^{* *_{e}}$ & $0,529^{* * e}$ & $0,330^{* e}$ & & 0,212 \\
\hline BAI & & $<24$ & 15,23 & 9,34 & $0,026^{e}$ & $0,516^{* * \mathrm{~s}}$ & $0,436^{* * e}$ & - & $0,461^{* * \mathrm{~s}}$ & \\
\hline$>23$ & & 15,19 & 10,29 & $0,063^{\mathrm{e}}$ & $0,529^{* * e}$ & $0,523^{* * \mathrm{e}}$ & - & $0,516^{* * \mathrm{e}}$ & & נונ, \\
\hline
\end{tabular}

Nota: $\mathrm{N}=335$; <24: de 18 a 23 años; >23: de 24 a 30 años; DE: Desviación estándar; a: Media y desviación estándar del rango en la escala BAI; r1: Correlación del criterio con la variable Edad; r2: Correlación del criterio con la variable Ansiedad subjetiva; r3: Correlación del criterio con la variable Depresión subjetiva; r4: Correlación del criterio con la prueba BAI; r5:Correlación del criterio con la prueba BDI; s: Coeficiente de correlación Rho de Spearman; e: Coeficiente de correlación de Pearson; $p$ : valor de significancia estadística de la comparación; U: Prueba estadística U de Mann-Whitney; *: $p \leq 0.05 ;{ }^{* *}: p \leq 0.01 ;{ }^{* * *}: p \leq 0.001$.

Los datos estadísticos de la tabla 5 indican que, de los participantes menores de 24 años, que asignaron puntuaciones para Ansiedad subjetiva por autopercepción muy baja (18.1\%) el 7.7 \% de estos cumplía realmente criterios 
para Ansiedad moderada en la Escala BAI y el porcentaje restante coincidía con el nivel de Muy baja ansiedad en esta escala objetiva. En contraste a lo anterior, el $100 \%$ de los mayores de 23 años, cuyas puntuaciones se ubicaron en el rango de muy baja ansiedad por autopercepción, fue coherente con las puntuaciones obtenidas para este mismo rango en la Escala BAI.

En la tabla 5, el 36.6 \% de los menores de 24 años puntuó Ansiedad subjetiva moderada, aunque sólo el 14.3 \% cumplió criterios de Ansiedad moderada en la Escala objetiva; comportamientos cercanos a este análisis fueron presentados por los mayores de 23 años para este mismo rango en autopercepción. De manera semejante, el $45.3 \%$ de los menores de 24 ańos y el $52.1 \%$ de los mayores de 23 ańos obtuvieron puntuaciones que los ubicaron en el rango de Ansiedad subjetiva Severa, aunque sólo el 6.9 \% y el $8 \%$ respectivamente cumplían criterios de Ansiedad Severa en el BAI. También se encontró que, de la muestra total, menos del 5\% cumplen criterios clínicos de Ansiedad Severa (BAI).

En relación con las comparaciones estadísticas por edad (tabla 5), se observó que, significativamente no existen diferencias entre los rangos presentados en la tabla, tanto para el criterio de Ansiedad subjetiva como para BAI. Sin embargo, en lo que respecta a las correlaciones, se halló que, los dos criterios, tanto para los $<24$ como para $>23$, se relacionan bidireccionalmente de forma directa ( $\mathrm{r} 4 \mathrm{y} \mathrm{r} 2$, respectivamente), indicando que, a mayores puntuaciones en el criterio, mayores puntuaciones en la escala relacionada $(p \leq 0.01)$. Ambos criterios se correlacionan con las escalas de depresión ( $\mathrm{r} 3$ y r5), aunque, para los $<24$ la significancia estadística con la escala BDI ( 5 ) se establece con valor de $p \leq 0.01$ y para los $>23$ a nivel de $p \leq 0.05$.

De forma general, en la tabla 6 se encontró que, entre otros aspectos, el $26.4 \%$ de los menores de 24 años asignaron puntuaciones en la escala de Depresión subjetiva por autopercepción que los ubicaron en el rango Moderado; pese a esto, sólo el $3.9 \%$ de ese porcentaje cumplió criterios de depresión Moderada en la Escala objetiva (BDI) y el 1.3 \% alcanzó el rango de depresión severa, mientras que los demás obtuvieron puntuaciones inferiores al nivel autopercibido. En lo que respecta a los mayores de 23 ańos, el rango de Depresión subjetiva Severa fue ocupado por el 14.6 \% de estos, aun cuando sólo el 
[78] Jorge Alexander Ríos, Carolina Escudero, Claudia Rocío López, Claudia Margarita Estrada, Jonathan Montes, Albeiro de Jesús Muñoz

$28.6 \%$ (del $14.6 \%$ ) se ubicó objetivamente en el rango de depresión Severa del BDI; ninguno cumplió criterios objetivos de depresión Moderada y el porcentaje restante se distribuyó en los niveles objetivos de depresión ausente, mínima y leve, representando este último la mitad del porcentaje que ocuparon los rangos anteriores.

También se encontró que de la muestra total (tabla 6), el mayor porcentaje de los participantes, sin importar la edad, estuvo ubicado en el rango de depresión minima (BDI). Resalta en la tabla que, de los mayores de 23 años, el 16.6 \% se ubicó en el nivel Leve de Depresión objetiva (Total BDI) y también el $16.6 \%$ de los mayores de 23 ańos obtuvo puntuaciones que los ubicó en el nivel leve de Depresión subjetiva por autopercepción, pese a esto, no fueron las mismas personas las que se ubicaron en estos porcentajes, puesto que ninguna persona del nivel leve de depresión por autopercepción (>23) cumplió criterios para nivel leve de Depresión objetiva (BDI).

Tabla 6. Coherencia entre Autopercepción de depresión y Depresión objetiva por edad

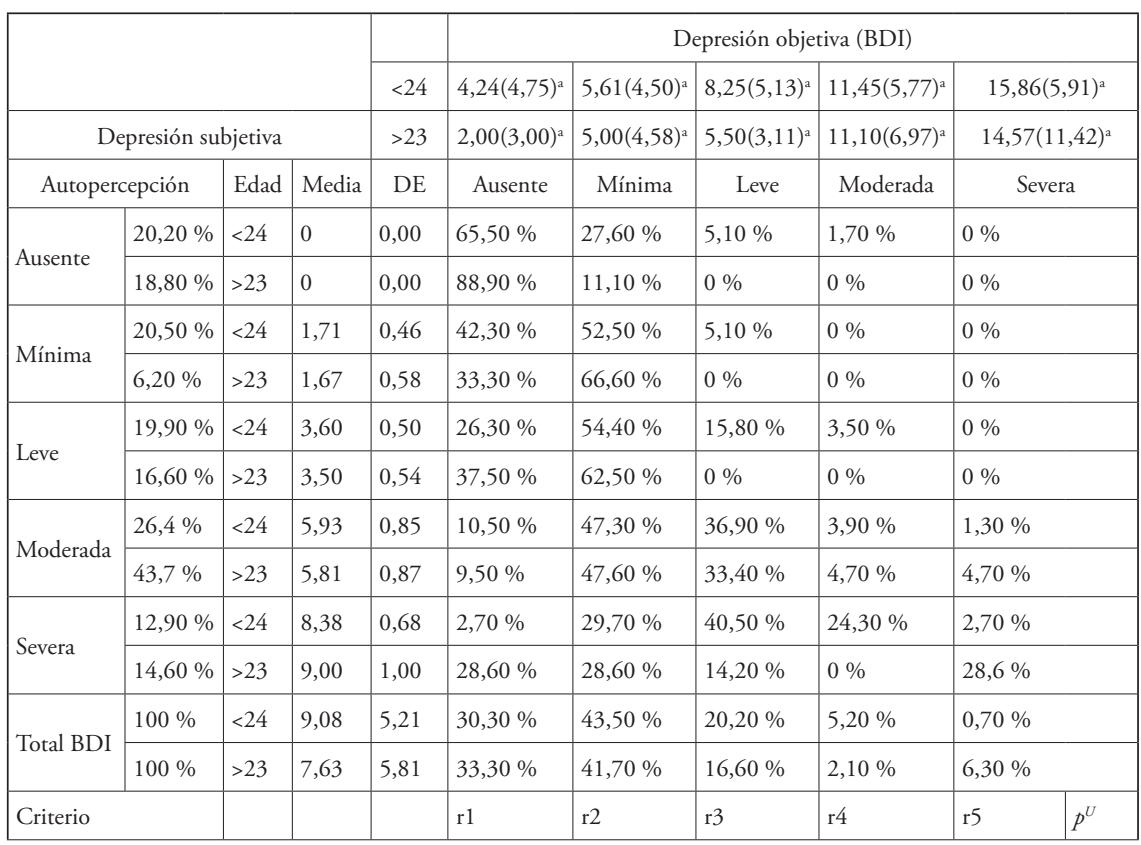


Autopercepción del estado de ánimo y presencia de ansiedad y depresión en estudiantes universitarios

\begin{tabular}{|l|l|l|l|l|l|l|l|l|l|}
\hline \multirow{2}{*}{$\begin{array}{l}\text { Depresión Subjetiva } \\
>23\end{array}$} & $<24$ & 3,72 & 2,85 & $-0,028^{s}$ & $0,438^{* * s}$ & - & $0,436^{* * e}$ & $0,611^{* * e}$ & \multirow{2}{*}{0,078} \\
\cline { 2 - 9 } & 4,54 & 2,98 & $0,091^{s}$ & $0,378^{* * e}$ & - & $0,523^{* * e}$ & $0,583^{* * e}$ & & \\
\hline \multirow{2}{*}{$\begin{array}{l}\text { BDI } \\
>23\end{array}$} & $<24$ & 8,72 & 6,45 & $0,012^{\text {s }}$ & $0,333^{* * s}$ & $0,611^{* * e}$ & $0,461^{* * s}$ & - & \\
\cline { 2 - 10 } & 8,58 & 7,75 & $0,047^{\mathrm{s}}$ & $0,330^{* e}$ & $0,583^{* * e}$ & $0,516^{* * e}$ & - & & 0,916 \\
\hline
\end{tabular}

Nota: $N=335$; <24: de 18 a 23 años; >23: de 24 a 30 años; DE: Desviación estándar; a: Media y desviación estándar del rango en la escala BDI; r1: Correlación del criterio con la variable Edad; r2: Correlación del criterio con la variable Ansiedad subjetiva; r3: Correlación del criterio con la variable Depresión subjetiva; r4: Correlación del criterio con la prueba BAI; r5:Correlación del criterio con la prueba BDI; s: Coeficiente de correlación Rho de Spearman; e: Coeficiente de correlación de Pearson; $p$ : valor de significancia estadística de la comparación; U: Prueba estadística U de Mann-Whitney; ${ }^{*}: p \leq 0.05 ;{ }^{* *}: p \leq 0.01{ }^{* * *}: p \leq 0.001$.

$\mathrm{Al}$ igual que con las escalas de ansiedad, estadísticamente se encontró que no hay diferencias entre los rangos de edad en los criterios de Depresión subjetiva y BDI (tabla 6), aunque las mayores puntuaciones en la escala de Depresión objetiva fueron obtenidas por los menores de 24 años en todos los rangos de la escala, al igual que en la escala de Depresión subjetiva por autopercepción, exceptuando el rango de depresión Severa, donde los mayores de 23 años asignaron en promedio mayores puntuaciones. Sin embargo, se estableció una correspondencia estadística entre estos dos criterios (Depresión subjetiva y BDI), encontrándose correlaciones bidireccionales, entre sí ( $\mathrm{r} 5$ y r3, respectivamente). Estas relaciones ( $p \leq 0.01)$ se presentan de forma directa y proporcional, señalando que cuando las puntuaciones en el criterio aumentan, también lo hacen las de la escala relacionada. Por otra parte, ambos criterios se correlacionaron con las escalas de ansiedad, tanto objetiva como subjetiva ( $\mathrm{r} 4$ y r2, respectivamente), resaltando que, para el caso de la relación con la escala subjetiva, el criterio BDI se obtiene una significancia con valor de $p \leq 0.05$ para el grupo de los mayores de 23 años y de $p \leq 0.01$ para los menores de 24 .

Los datos descritos anteriormente permitieron establecer análisis estadísticos a nivel general y comparativo que denotan el grado de coherencia (mayor o menor) entre los estados emocionales valorados por autopercepción y los resultados de las escalas estandarizadas empleadas para la evaluación objetiva de dichos estados, por lo que se destaca que existe correlación interna entre las escalas subjetivas y objetivas, y coherencia variable entre la autopercepción y el cumplimiento de los criterios clínicos establecidos en las escalas objetivas. En consideración a esto, los análisis cualitativos que describen funcionalmen- 
te los análisis cuantitativos aquí descritos se plantearán a profundidad en los apartados siguientes de discusión y conclusiones.

\section{Discusión}

Los resultados obtenidos a partir de los análisis realizados han permitido responder al cuestionamiento base de esta investigación; encontrándose que los estudiantes universitarios presentan dificultades en la habilidad que se requiere para reconocer asertivamente los estados de ánimo propios, con limitaciones de autopercepción para valorar la magnitud real de estos. Lo que devela falencias en el empoderamiento y desarrollo adecuado de la inteligencia emocional y en la identificación oportuna de los momentos en que se requiere dar atención a la salud mental.

En este sentido, aun cuando los estudios desarrollados en población universitaria han sido limitados en extensión metodológica para el contexto Latinoamericano, en Colombia se han llevado a cabo algunos que ofrecen datos relevantes para su análisis y discusión, sumando a esto los datos ofrecidos por la OMS y los estudios de salud mental de las poblaciones en general, donde, los estados de ánimo considerados aquí, presentan relaciones importantes a considerar en los planes y programas de promoción de la salud y prevención de la enfermedad mental, encontrándose ampliamente que cada vez son más las patologías psiquiátricas que presentan sus primeras fases de desarrollo y evolución en periodos de adolescencia y adultez joven.

Por su parte, los datos presentados por la Universidad Industrial de Santander indicaron que el $9 \%$ de los estudiantes atendidos presentó un trastorno de ansiedad (UIS, 2005), y aunque esta institución no refiere el nivel de sintomatología presentado por los estudiantes, en contrapartida, nuestra investigación encontró que el $21.8 \%$ de los universitarios que hicieron parte de la muestra presentó ansiedad moderada y 3.2 \% cumplió criterios clínicos de ansiedad severa.

Lo anterior contrasta con el $17 \%$ de la población que presentó rasgos de ansiedad en la Universidad Pontificia Bolivariana de Bucaramanga (UPB, 2005), aun cuando no fue especificado el grado de sintomatología presentado. 
Sin embargo, el porcentaje de estudiantes que presentó algún nivel de ansiedad, en el estudio aquí desarrollado y ya descrito fue de $25 \%$; porcentaje que es inferior al 76.2\% reportado por Arrieta et al. (2013), en universidades de Cartagena. Asimismo, el porcentaje de estudiantes con algún grado de ansiedad de nuestra investigación también es inferior al encontrado en el estudio desarrollado con estudiantes por Cardona-Arias et al. (2015) en Medellín, donde la prevalencia de ansiedad fue del $58 \%$.

Entre tanto, Balanza, Moreno y Guerrero (2009), identificaron que el 19.3 $\%$ de la población de su estudio, presentó en algún momento un trastorno de ansiedad más habitual en mujeres. En relación con esto, la investigación aquí ejecutada halló que el $23.5 \%$ de las mujeres presentó niveles entre ansiedad moderada y severa; y aunque los datos de Balanza no mencionan la prevalencia en hombres, aquí se encontró que el $29 \%$ de estos presentó niveles altos de ansiedad, cumpliendo el $3.2 \%$ criterios clínicos de ansiedad severa. Nuestro estudio encontró, además, que de forma objetiva existe una distribución estadística y clínica similar entre hombres y mujeres, y por edades, esto coincide con lo formulado por Cardona-Arias et al. (2015) en su estudio sobre la prevalencia de ansiedad entre universitarios.

Tal como lo mencionaron Murray et al. (2012) y Ferrari et al. (2013), el trastorno depresivo se ha catalogado como una de las principales patologías mentales más discapacitante del mundo, en consideración a esto, el ministerio de salud en Colombia (MINSALUD, 2017), presentó datos de la OMS en los cuales se reportó que cerca del $4.7 \%$ de la población presentó sintomatología depresiva. Nuestra investigación encontró que el 70\% de los estudiantes cumple criterios clínicos significativos de depresión y aunque no es posible atribuir las causas de estos índices, diversas investigaciones en Colombia (Hurtado et al., 2011; Kuehner, 2003; Restrepo et al., 2018), han sugerido que uno de los factores clave está asociado a la predisposición de los jóvenes universitarios al consumo de sustancias psicoactivas, disfunciones familiares y problemáticas socio-económicas.

En Colombia, también se ha indicado que las mujeres y los jóvenes presentan mayores índices de depresión (MINSALUD, 2017), y aun cuando los estudios no presentan datos puntuales, la investigación aquí desarrollada en- 
contró que cerca del $70 \%$ de las mujeres presenta algún nivel de depresión y que el $6.2 \%$ de las estudiantes cumplió criterios clínicos de depresión moderada y severa. Por otra parte, estos estudios no mencionan la prevalencia en hombres, ante lo cual aquí se encontró que cerca del $60 \%$ de estos presenta sintomatología depresiva y que el $3.2 \%$ corresponde a depresión severa y el mismo porcentaje para niveles de depresión moderada. Se encontró también que los menores de 24 años presentan índices significativos de depresión siendo cerca del $70 \%$ de los estudiantes. Con estos datos es posible concordar con lo postulado por Pérez-Álvarez y García-Montes (2001), al referir que esta patología altamente incapacitante pese a manifestarse en cualquier momento de la vida, suele tener una mayor incidencia en la adolescencia.

Ante este panorama se hace factible plantear la relación existente entre manifestaciones clínicas de ansiedad y depresión, y la valoración subjetiva de estos estados; con esto, se hace posible, como fue planteado en diversas investigaciones (Azzam, Chandavarkar \& Mathews, 2007; Barraza et al., 2015; Barraza-López, Muñoz-Navarro y Behrens-Pérez, 2017; Delgado-Gómez et al., 2019; Gómez-Romero et al., 2018), expresar la existencia de una alta correlación de los estados de ansiedad y depresión y la autopercepción emocional negativa, tal como lo sugieren los datos obtenidos en nuestro estudio. Asimismo, es viable coincidir con Agudelo, Casadiegos y Sánchez (2008; 2009), al aseverar que entre los jóvenes universitarios existe una asociación significativa entre la ansiedad y la depresión; llegando a ser posible sugerir, como lo han hecho Barraza-López Muñoz-Navarro y Behrens-Pérez (2017), que estas correlaciones pueden ser la explicación al modo en que los altos niveles de depresión y ansiedad estarían implicados en la tendencia de los estudiantes a presentar ciclos de pensamientos rumiativos que intensifica las autopercepciones negativas y aumenta la presencia de manifestaciones clínicas de consideración.

\section{Conclusiones}

Los hallazgos de la investigación aquí desarrollada suponen aportes significativos y relevantes para el afrontamiento y desarrollo de programas de intervención de los departamentos de bienestar universitario y de tratamientos 
por psicología orientados a la atención de adolescentes y adultos jóvenes, posibilitando formular estrategias de empoderamiento y de los propios estados emocionales, para favorecer el fortalecimiento de la inteligencia emocional en consideración al periodo crítico de desarrollo neurobiológico en que se encuentra esta población.

Los resultados permitieron determinar que existe correspondencia entre la valoración subjetiva, por autopercepción del estado de ánimo, con la medición objetiva realizada por medio de la implementación de pruebas psicométricas validadas para la medición de la sintomatología característica de ansiedad y depresión. En consecuencia, a mayor sensación de estados depresivos y ansiosos por parte de los participantes, mayores puntuaciones eran obtenidas en las pruebas estandarizadas.

Los participantes se autopercibieron, de forma general, con estados de ansiedad moderada con tendencias a niveles de ansiedad severa y manifestaron sentirse con depresión de nivel leve, la cual oscila entre síntomas mínimos y moderados de este estado. Sin embargo, el nivel real de ansiedad presentado por los estudiantes se encontró en el rango muy bajo en las convenciones de la escala, y de forma general cumplieron criterios clínicos para depresión mínima.

En lo que respecta a las diferencias por género entre los estudiantes universitarios, las mujeres se autoperciben con mayores niveles de ansiedad y depresión, reportando mayor sensación de estados depresivos en comparación con los hombres. Pese a esto, se halló que tanto hombres como mujeres presentaron objetivamente niveles similares de ansiedad y depresión según las escalas psicométricas.

En lo que respecta a la valoración de ansiedad, la mayoría de los estudiantes se autopercibieron con niveles de ansiedad severa. Sin embargo, menos del $4 \%$ de estos cumplió realmente criterios objetivos para niveles severos de ansiedad y el $75 \%$ presentó realmente niveles muy bajos de ansiedad. En la mayoría de los casos los estudiantes atribuyeron subjetivamente mayores niveles que los verdaderamente presentados.

De forma particular, las mujeres manifestaron sensaciones que las ubicarían en criterios de ansiedad severa. Sin embargo, las medidas objetivas las 
situaron en niveles de ansiedad muy baja al igual que a los hombres, quienes se autopercibieron con ansiedad moderada. Por su parte, en los niveles bajos de la escala son los hombres quienes manifiestan una mayor sensación de ansiedad, aunque objetivamente, son las mujeres quienes cumplen mayores criterios para estos rangos.

En referencia a los estados de depresión se encontró que, la sensación de los estudiantes, aun cuando se autopercibieron con mayores niveles que los presentados realmente, tiene una mayor cercanía con los valores de las escalas objetivas; y se halló que algunos de los estudiantes que manifestaron subjetivamente no sentir depresión cumplían realmente criterios clínicos de depresión leve o moderada. De forma general, más de la mitad de los estudiantes no presentó coherencia entre los niveles de depresión manifestados y los objetivamente presentados.

De un lado, entre los participantes, tanto mujeres como hombres se autopercibieron con niveles de depresión leve, aunque las mujeres presentaron tendencia a niveles moderados y los hombres a niveles mínimos de depresión. Pese a lo anterior, ambos géneros, en promedio, cumplieron objetivamente criterios clínicos de depresión mínima. Para los niveles críticos de depresión se encontró que el doble de mujeres con relación a los hombres presenta depresión moderada y tres veces más los hombres que las mujeres presentan realmente depresión severa.

De otro lado, al dividir por grupos de edad a los estudiantes universitarios, se encontró que no hay diferencias clínicas entre los mayores de 23 y los menores de 24 años en lo que respecta a la autopercepción del estado de ánimo y los resultados de la valoración objetiva para la sintomatología característica de ansiedad y depresión.

Por su parte, en relación con la coherencia entre la autopercepción de ansiedad y la ansiedad objetiva, los estudiantes mayores de 23 años dieron cuenta de manera más aproximada de los niveles reales de ansiedad que presentaban. Cerca de la mitad de los estudiantes sin importar su edad manifestaron niveles de ansiedad severa, sin embargo, más del $70 \%$ de estos presentaba objetivamente ansiedad muy baja. 
En los niveles bajos de depresión, los estudiantes no presentaron coherencia entre la autopercepción del estado de ánimo y la depresión objetiva, puesto que, un porcentaje significativo presentó niveles mayores a los autopercibidos. Son los estudiantes mayores de 23 ańos los que reportan subjetivamente niveles de depresión moderada y severa, encontrándose que objetivamente cumplen criterios para estos niveles, más que los menores de 24 años.

En este estudio se encontraron discrepancias tanto por género como por rango de edad entre la autopercepción de los estados de ánimo y los niveles reales que presentan los estudiantes universitarios, lo cual da cuenta de la dificultad de reconocer sus propios estados en los que, para ansiedad suponen presentar mayores niveles, y para depresión autoreconocen menores criterios de los que objetivamente se tendrían en cuenta para atribuir un rango clínico a esta sintomatología. Lo anterior permite plantear cuestionamientos en cuanto a las herramientas psicológicas que poseen los estudiantes universitarios para el manejo y afrontamiento de las características emocionales y sociales de las que depende la inteligencia emocional, así como el uso y la lectura asertiva de ésta.

\section{Referencias}

Agudelo, D., Casadiegos, C., y Sánchez, D. (2008). Características de ansiedad y depresión en estudiantes universitarios. International Journal of Psychological Research, 1(1), 34-39. Recuperado de http://www.redalyc.org/articulo. oa?id=299023503006

Agudelo, D., Casadiegos, C., y Sánchez, D. (2009). Relación entre esquemas maladaptativos tempranos y características de ansiedad y depresión en estudiantes universitarios. Universitas Psychologica, 8(1), 87-104. Recuperado de http:// www.redalyc.org/articulo.oa?id=64712168008

American Psychological Association. (2010). Trastornos de la ansiedad: el papel de la psicoterapia en el tratamiento eficaz. Washington: Ed. APA. Recuperado de http://www.apa.org/centrodeapoyo/tratamiento.aspx

Arrieta, V, Díaz, C., y González, M. (2013). Síntomas de depresión, ansiedad y estrés en estudiantes de odontología: prevalencia y factores relacionados. Revista Colombiana de Psiquiatría, 42(2), 173-181. Recuperado de http://www.redalyc. org/articulo.oa?id=80629187004 
[86] Jorge Alexander Ríos, Carolina Escudero, Claudia Rocío López, Claudia Margarita Estrada, Jonathan Montes, Albeiro de Jesús Muñoz

Azzam, A., Chandavarkar, U., \& Mathews, C. (2007). Anxiety symptoms and perceived performance in medical students. Depress Anxiety, 24(2), 103-11. doi: $10.1002 /$ da. 20185

Balanza, S., Morales, I., y Guerrero, J. (2009). Prevalencia de Ansiedad y Depresión en una Población de Estudiantes Universitarios: Factores Académicos y Sociofamiliares Asociados. Clínica y Salud, 20(2), 177-187. Recuperado de http://scielo.isciii.es/scielo.php?script=sci_arttext\&pid=S1130-52742009000200006\&l$\mathrm{ng}=\mathrm{es} \& \mathrm{t} \operatorname{lng}=\mathrm{pt}$.

Bar-On, R. (1997). Bar-On Emotional Quotient Inventory (EQ-i): Technical manual. Toronto, Canadá: Multi-Health Systems.

Barraza, A., y Medina, S. (2016). El estrés académico en estudiantes de gastronomía de una universidad privada de la ciudad de Durango, en México. Revista de Psicología Universidad de Antioquia, 8(2), 11-26. doi: 10.17533/udea.rpsua. $\mathrm{v} 8 \mathrm{n} 2 \mathrm{a} 02$

Barraza, R., Muñoz, N., Alfaro, M., Álvarez, A., Araya, V., Villagra, J., y Contreras, A. (2015). Ansiedad, depresión, estrés y organización de la personalidad en estudiantes novatos de medicina y enfermería. Revista Chilena de neuropsiquiatría, 53(4), 251-60. doi: 10.4067/S0717-92272015000400005

Barraza-López, R., Muñoz-Navarro, N., y Behrens-Pérez, C. (2017). Relación entre inteligencia emocional y depresión-ansiedad y estrés en estudiantes de medicina de primer ańo. Revista chilena de neuropsiquiatría, 55(1), 18-25. doi: 10.4067/ S0717-92272017000100003

Beck, A. (2002). Inventario de ansiedad de BECK. México: Pearson Clinical.

Beck, A. (2002a). Inventario de depresión de BECK-II. México: Manual Moderno.

Bisquerra, E., Punset, E., Mora, F., García, E., López-Cassá, E., Pérez-González, J.C., y Planllis, O. (2012). ¿Cómo educar las emociones? La inteligencia emocional en la infancia y la adolescencia. Esplugues de Llobregat (Barcelona): Hospital Santa Joao de Déu.

Bisquerra, R. (2006). Orientación psicopedagógica y educación emocional. Estudios sobre Educación, 11, 9-25. Recuperado de http://dadun.unav.edu/bitstream/10171/9208/1/Ea.pdf

Botelho, S., Martínez, L., Conde, C., Prada, E., y Bezerra, C. (2004). Evaluación de la memoria declarativa asociada a contenido emocional en una muestra colombiana. Revista Latinoamericana de psicología, 36(2), 229-242. Recuperado de www. redalyc. org/pdf/805/80536204.pdf 
Autopercepción del estado de ánimo y presencia de ansiedad y depresión en estudiantes universitarios

Caballero, C., Breso, E., y González, O. (2015). Burnout en estudiantes universitarios. Psicología desde el Caribe, 32(3), 424-441. Recuperado de https://www. redalyc.org/pdf/213/21342681007.pdf

Campo-Arias, A., Díaz, L., Rueda-Jaimes, G., y Barros, J. (2005). Validación de la escala de Zung para depresión en universitarias de Bucaramanga, Colombia. Revista Colombiana de psiquiatría, 34, 54-62. Recuperado de http://www.redalyc. org/articulo.oa?id=80628403005

Campo-Cabal, G., y Gutiérrez, J. (2001). Psicopatología en estudiantes universitarios de la facultad de salud, Univalle. Revista Colombiana de Psiquiatría, 30(4), 351358. Recuperado de http://www.redalyc.org/pdf/806/80630402.pdf

Cardona-Arias, J., Pérez-Restrepo, D., Rivera-Ocampo, S., Gómez-Martínez, J., y Reyes, A. (2015). Prevalencia de ansiedad en estudiantes universitarios. Revista Diversitas: Perspectivas en Psicología, 11(1), 79-89. Recuperado de http://www. scielo.org.co/pdf/dpp/v11n1/v11n1a06.pdf

Cruzblanca, H., Lupercio, P., Collas, J., y Castro, E. (2016). Neurobiología de la depresión mayor y de su tratamiento farmacológico. Salud mental, 39(1), 47-58. doi: http://dx.doi.org/10.17711/SM.0185-3325.2015.067

Cupul-García, J., Hinojosa-Calvo, E., Villa-Rodríguez, M., Herrera-Guzmán, I., y Padrós-Blázquez, F. (2018). Evaluación neuropsicológica básica para adultos en pacientes con trastorno de ansiedad generalizada. Revista chilena de neuropsiquiatría, 56(3), 151-160. Doi: 10.4067/s0717-92272018000300151

Dávila, F., Ruiz, C., Moncada, A., y Gallardo, R. (2011). Niveles de ansiedad, depresión y percepción de apoyo social en estudiantes de Odontología de la Universidad de Chile. Revista de Psicología, 20(2), 147-172. Recuperado de http://www. redalyc.org/articulo.oa?id=26421338007

Delgado-Gómez, M., Gómez-Díaz, M., Gómez-Sánchez, R., y Reche-García, C. (2019). Relación entre Inteligencia Emocional y Riesgo Psicopatológico en Estudiantes Universitarios. Formación universitaria, 12(3), 39-46. doi: 10.4067/ S0718-50062019000300039

Fernández, C., y Giraldo, H. (2001). Prevalencia del trastorno depresivo y factores asociados a esta patología en los estudiantes de pregrado del programa de Medicina de la Universidad de Caldas, en el segundo semestre del año 2000. Manizales: Universidad de Caldas, Facultad de Ciencias para la Salud, Departamento de Salud Mental y Comportamiento Humano. Recuperado de: http://www.scielo.org.co/scielo.php?script=sci_nlinks\&ref=000115\&pi$\mathrm{d}=$ S0034-7450200300040000300006\&lng= 
Fernández-Berrocal, P., Salovey, P., Vera, A., Extremera, N., \& Ramos, N. (2005). Cultural influences on the relation between perceived emotional intelligence and depression. International Review of Social Psychology, 18, 91-107. Recuperado de http://emotional.intelligence.uma.es/documentos/PDF2emotional_intelligence_and_depression.pdf

Ferrari, A., Charlson, F., Norman, R., Patten, S., Freedman, G., Murray, C.J., \& Whiteford, H. (2013). Burden of depressive disorders by country, sex, age, and year: findings from the global burden of disease study 2010. Journal PLoS medicine, 1O(11), e1001547. doi: 10.1371/journal.pmed.1001547

Flores, R., Jiménez, S., Pérez, S., Ramírez, P., y Vega, C. (2007). Depresión y Ansiedad en Estudiantes Universitarios. Revista Electrónica de Psicología Iztacala, 10(2), 94-105. Recuperado de http://www.iztacala.unam.mx/carreras/psicologia/psiclin/vol10num2/art6V10N2jun07.pdf

Fried, E., \& Nesse, R. (2015). Depression is not a consistent syndrome: An investigation of unique symptom patterns in the STAR*D study. Journal Affect Disord, 172: 96-102. Doi: 10.1016/j.jad.2014.10.010

Gallagher, R. (2002). Nacional Survey of Counselling Center Directors. Pittsburg: INT Associations of Counselling Services INC. Recuperado de http://d-scholarship. pitt.edu/28162/1/counseling_center_survey_023.pdf

Galli, A. (2005). Prevalencia de trastornos psicopatológicos en alumnos de psicología. Revista de psiquiatría y salud mental Hermilio Valdizan, 6(1), 55-66. Recuperado de http://www.hhv.gob.pe/revista/2005/6\%20PREVALENCIA\%20DE\%20 TRASTORNOS\%20PSICOPATOLOGICOS.pdf

Goleman, D. (1996). Inteligencia emocional. Barcelona: Editorial Kairós.

Goleman, D. (2013). El cerebro y la inteligencia emocional. Barcelona: S.A. Ediciones B.

Goleman, D., y Boyatzis, R. (2008). La inteligencia social y la biología del liderazgo. Harvard Business Review, 86(9), 86-95. Recuperado de: https://dialnet.unirioja. es/servlet/articulo?codigo $=2704182$

Gómez-Romero, M., Limonero, J., Toro, J., Montes-Hidalgo, J., y Tomás-Sábado, J. (2018). Relación entre inteligencia emocional, afecto negativo y riesgo suicida en jóvenes universitarios. Ansiedad y Estrés, 24(1), 18-23. doi: 10.1016/j. anyes.2017.10.007

González-Forteza, C., Hermosillo, A., Vacio-Muro, M., Peralta, R., y Wagner, F. (2015). Depresión en adolescentes. Un problema oculto para la salud pública y la práctica clínica. Boletín médico del Hospital Infantil de México, 72(2), 149155. doi: 10.1016/j.bmhimx.2015.05.006 
Autopercepción del estado de ánimo y presencia de ansiedad y depresión en estudiantes universitarios

Hernández, R., Fernández, L., y Baptista, P. (2014). Metodología de la investigación. 6ta Ed Mexico: McGrawHill.

Hurtado, H., Sánchez, V., Bedoya, L., Londoño, J., Marín, C., Muñíz, O., Agudelo, D., y Gómez, Y. (2011). Prevalencia de trastornos mentales en usuarios de consulta psicológica en una universidad colombiana. Revista de Psicología Universidad de Antioquia, 3(1), 59-71. Recuperado de http://pepsic.bvsalud.org/scielo. php?script=sci_arttext\&pid=S2145-48922011000100005

Jiménez, M., y López-Zafra, E. (2009). Inteligencia emocional y rendimiento escolar: estado actual de la cuestión. Revista Latinoamericana de Psicología, 41(1), 69-79. Recuperado de http://www.redalyc.org/pdf/805/80511492005.pdf

Jiménez, M., y López-Zafra, E. (2014). Actitudes sociales y adaptación social en adolescentes españoles: el papel de la inteligencia emocional percibida. Revista de Psicología Social, 26(1), 105-117. doi: 10.1174/021347411794078417

Kuehner, C. (2003). Gender differences in unipolar depression: An update of epidemiological findings and possible explanations. Revista Acta Psychiatr Scand, 108(3), 163-174. Recuperado de https:/www.ncbi.nlm.nih.gov/pub$\mathrm{med} / 12890270$

Ley 1090 (2006). Por la cual se reglamenta el ejercicio de la profesión de Psicología, se dicta el Código Deontológico y Bioético y otras disposiciones. Congreso de Colombia. Bogotá: Ministerio de protección social.

Maitta, I., Cedeño, M., y Escobar, M. (2018). Factores biológicos, psicológicos y sociales que afectan la salud mental. Revista Caribeña de Ciencias Sociales. Recuperado de https://www.eumed.net/rev/caribe/2018/03/factores-salud-mental.html

Marcus, M., Yasamy, M., Van Ommeren, M., Chisholm, D., \& Saxena, S. (2012). A global public health concern. OMS. Recuperado de http://www.who.int/mental_health/management/depression/who_paper_depression_wfmh_2012.pdf

Marenco, A., Suárez, Y., y Palacio, J. (2017). Burnout académico y síntomas relacionados con problemas de salud mental en universitarios colombianos. Psychologia. Avances de la disciplina, 11(2), 45-55. Recuperado de http://www.scielo.org. co/pdf/psych/v11n2/1900-2386-psych-11-02-00051.pdf

Mayer, J., \& Salovey, P. (1997). What is emotional intelligence? En P. Salovey, \& D. J. Sluyter (Eds.), Emotional development and emotional intelligence: Educational implications (pp. 3-34). New York: Harper Collins.

Minoletti, A., Sepúlveda, R., \& Horvitz-Lennon, M. (2012). Twenty Years of Mental Health Policies in Chile: Lessons and Challenges. Journal Int J Ment Health, 
[90] Jorge Alexander Ríos, Carolina Escudero, Claudia Rocío López, Claudia Margarita Estrada, Jonathan Montes, Albeiro de Jesús Muñoz

41(1), 21-37. Recuperado de http:/www.tandfonline.com/doi/abs/10.2753/ IMH0020-7411410102

MINSALUD. (2017). Boletín de salud mental Depresión Subdirección de Enfermedades No Transmisibles. Ministerio de la salud y la protección, Colombia. Recuperado de https:/www.minsalud.gov.co/sites/rid/Lists/BibliotecaDigital/ RIDE/VS/PP/ENT/boletin-depresion-marzo-2017.pdf

Murray, C., Vos, T., Lozano, R., Naghavi, M., Flaxman, A., Michaud, C., \& Ross, A. (2012). Disability-adjusted life years (DALYs) for 291 diseases and injuries in 21 regions, 1990-2010: a systematic analysis for the Global Burden of Disease Study 2010. Journal The Lancet, 380(9859), 2197-2223. doi: 10.1016/S01406736(12)61689-4

Pardo, G., Sandoval, A., y Umbarila, D. (2004). Adolescencia y depresión. Revista Colombiana de Psicología, 13, 13-28. Recuperado de https://revistas.unal.edu. co/index.php/psicologia/article/view/1204/1755

Peláez, L., Londoño, A., Gartner, C., Agudelo, C., Martínez, L., Tirado, A., y Ortiz, I. (2014). Eventos estresores y factores psicosociales en estudiantes de una universidad privada de Medellín, Colombia. Revista de Psicología Universidad de Antioquia, 6(2), 111-120. Recuperado de https://aprendeenlinea.udea.edu.co/ revistas/index.php/psicologia/article/view/23386

Pérez-Álvarez, M., y García-Montes, J. (2001). Tratamientos psicológicos eficaces para la depresión. Psicothema, 13(3), 493-510. Recuperado de http://www.psicothema.com/pdf/471.pdf

Pérez-Padilla, E., Cervantes-Ramírez, V., Hijuelos-García, N., Pineda-Cortés, J., y Salgado-Burgos, H. (2017). Prevalencia, causas y tratamiento de la depresión Mayor. Revista de Biomedicina, 28, 89-115. Recuperado de http://www.medigraphic.com/pdfs/revbio/bio-2017/bio172c.pdf

Pineda, C. (2012). Inteligencia emocional y bienestar personal en estudiantes universitarios de ciencias de la salud. (Tesis doctoral). Málaga: Universidad de Málaga. Recuperado de https://riuma.uma.es/xmlui/bitstream/handle/10630/5384/ TDR_PINEDA_GALAN.pdf? sequence $=1$ \&isAllowed $=y$

Posada-Villa, J., Buitrago-Bonilla, J., Medina-Barreto, Y., y Rodríguez-Ospina, M. (2006). Trastornos de ansiedad según distribución por edad, género, variaciones por regiones, edad de aparición, uso de servicios, estado civil y funcionamiento/discapacidad según el Estudio Nacional de Salud Mental-Colombia. Revista NOVA Publicación Científica en Ciencias Biomédicas, 4(6), 33-41. Recuperado de: http://www.unicolmayor.edu.co/invest_nova/NOVA/NOVA6_ARTORIG2.pdf 
Autopercepción del estado de ánimo y presencia de ansiedad y depresión en estudiantes universitarios

Resolución No. 8430 de 1993. (2003). Normas Científicas, Técnicas y Administrativas para la Investigación en Salud. Ministerio de Salud, Colombia.

Restrepo, J., Amador Sánchez, O., Calderón, G., Castañeda, T., Osorio, Y., y Diez, P. (2018). Depresión y su relación con el consumo de sustancias psicoactivas, el estrés académico y la ideación suicida en estudiantes universitarios colombianos. Health and Addictions, 18(2), 227-239. Recuperado de https://dialnet.unirioja. es/servlet/articulo?codigo $=6546342$

Ríos-Flórez, J., y Flórez-Barco, E. (2017). Teoría de la mente en niños de 6 a 10 años de edad con antecedente de nacimiento prematuro y en edad escolar. Psychologia, 11(2), 29-43. doi: 10.21500/19002386.2626

Roca, M., Vives, M., López-Navarro, E., García-Campayo, J., y Gili, M. (2015). Alteraciones cognitivas y depresión: una revisión crítica. Actas Españolas de Psiquiatría, 43(5), 187-93. Recuperado de https://actaspsiquiatria.es/repositorio/17/97/ESP/17-97-ESP-187-93-619184.pdf

Rodríguez, A. (2013). Proyecto educativo de inteligencia emocional para educación infantil. ¿son compatibles el saber y el sentir? (Trabajo de grado), Universidad internacional de La Rioja, España. Recuperado de http://reunir.unir.net/handle/123456789/1887

Ruiz, A., Extremera, N., \& Pineda, G. (2014). Emotional intelligence, life satisfaction and subjective happiness in female student health professionals: The mediating effect of perceived stress. Journal of Psychiatric Mental Health Nursing, 21(2), 106-113. doi: 10.1111/jpm.12052

Salovey, P., \& Mayer, J. (1990). Emotional Intelligence. Imagination, Cognition, and Personality, 9(3), 185-211. Recuperado de http://journals.sagepub.com/doi/ abs/10.2190/DUGG-P24E-52WK-6CDG

Sequeira, A., y Fornaguera, J. (2014). Diferencias individuales en modelos animales: un enfoque para el estudio de factores neurobiológicos relacionados con depresión. Actualidades en Psicología, 28(117), 53-66. doi: 10.15517/ap. v28i117.14115

Torales, J., Arce, A., Riego, V., Chávez, E., Villalba-Arias, J., y Ruiz-Díaz, C. (2015). Psicofarmacología de la ansiedad. Tendencias en Medicina, 26(46), 79-86. Recuperado de http://tendenciasenmedicina.com/uploads/e7c12c712ba0645a545fef4e2db4b672.pdf

Torterolo, P., Scorza, C., Urbanavicius, J., Devera, A., Benedetto, L., Pascovich, C., Lagos, P., Chase, M., y Monti, J. (2014). Avances en el estudio de la neurobiología de la depresión: rol de la hormona concentradora de melanina. Revista 
[92] Jorge Alexander Ríos, Carolina Escudero, Claudia Rocío López, Claudia Margarita Estrada, Jonathan Montes, Albeiro de Jesús Muñoz

Médica del Uruguay, 30(2), 128-136. Recuperado de http://www.scielo.edu.uy/ scielo.php?pid=S1688-03902014000200008\&script=sci_arttext\&tlng=en

Universidad Industrial de Santander (UIS), Departamento de Bienestar Universitario (2005). Reporte de consultas atendidas según trastorno y/o diagnóstico establecido en el año 2004. Departamento de Bienestar Universitario, Universidad Industrial de Santander (UIS), Bucaramanga. Documento no publicado.

Universidad Pontificia Bolivariana (UPB), Departamento de Bienestar Universitario. (2005). Reporte de consultas atendidas según trastorno y/o diagnóstico establecido en los meses de junio a septiembre del año 2005. Departamento de Bienestar Universitario, Universidad Pontificia Bolivariana, Bucaramanga. Documento no publicado.

World Health Organization [WHO]. (2007). World Health Statistics [Internet]. Recuperado de http://www.who.int/gho/publications/world_health_statistics/ whostat2007.pdf

World Medical Association [WAM]. (2015). Declaración de Helsinki: Principios éticos para las investigaciones médicas en seres humanos. Declaración adaptada por el secretario de la WAM, tras emitirla en la $64^{\mathrm{a}}$ asamblea general [octubre 2013]. Fortaleza, Brasil. 